\title{
ATLANTIC and beyond: an interview with Professor Azfar Zaman
}

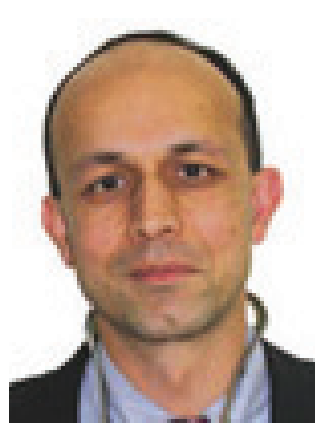

Professor Azfar Zaman* speaks to Wing Wu, Commissioning Editor: Professor Azfar Zaman is a Consultant Interventional Cardiologist at Freeman Hospital and Professor of Cardiology at Newcastle University. Following graduation at Leeds Medical School, he completed postgraduate training in cardiology at regional centres in Leeds, London and Cardiff. Prior to his appointment in Newcastle upon Tyne, he was a Fulbright Scholar and British Heart Foundation International Fellow at Mount Sinai Medical Center, New York, USA. He is the Clinical Lead for Coronary Intervention and Director of the Cardiac Catheter Laboratories. In 2012, he was appointed Specialty Group Lead for Cardiovascular Research and has an interest in clinical research, with a particular interest in atherothrombosis in diabetes and clinical trials.

\section{Q Can you tell us about your career background to date?}

I completed postgraduate training in cardiology at regional centers in Leeds, London and Cardiff, following which I was appointed a Fulbright Scholar and British Heart Foundation International Fellow at Mount Sinai Medical Center, New York, USA. I was appointed to the post of consultant interventional cardiologist at Freeman Hospital, Newcastle-upon-Tyne, UK, in 2001 and was awarded a personal chair in 2013. I am the Clinical Lead for Coronary Intervention and Director of the Cardiac Catheter Laboratories in a center performing $>2800$ percutaneous coronary interventions (PCIs) and $>60$ transcatheter aortic valve implantations annually.

\section{Q What first interested you in pursuing cardiology as a career path?}

From an early stage in my medical career, it was apparent that cardiovascular disease was a major health burden both in the UK and globally, responsible for increasing morbidity and mortality. Consequently, it was an active and exciting field for medical research leading to the rapid development of successful treatment options. In addition, cardiology had the option of being a procedure-based specialty with immediate patient benefits. For all of these reasons, cardiology had an early and lasting appeal, and I have to say, it has not disappointed!

\section{Q What are the current problems with oral antiplatelet treatment?}

For someone brought up in the era of aspirin, there are no problems - only benefits! Improvements in ischemic outcomes after acute coronary syndromes are testament to this and other classes of drugs. The problem is less with the antiplatelet drugs and more

*Freeman Hospital \& Newcastle University, Newcastle-upon-Tyne, UK; azfar.zaman@nuth.nhs.uk

Future $\because \cdots$ Medicine ${ }_{\text {part of }}$ 
with the patient population we are increasingly treating - and that is the elderly. This is a segment of the population that is growing worldwide and while they have the most to gain from more powerful oral agents, bleeding complications have the potential to negate these gains, if they are not used with caution.

\section{Can you tell us about ticagrelor?}

Ticagrelor is a second-generation antiplatelet agent that is more potent and has been shown to be more clinically effective in reducing ischemic events than clopidogrel in patients with acute coronary syndromes. It is approved in the UK (by NICE) for use in all patients with acute coronary syndromes.

\section{Q What are the benefits of using ticagrelor in comparison with other oral antiplatelet treatments?}

Being more potent than clopidogrel, against which it was compared in the PLATO trial, ticagrelor reduces ischemic events for up to 12 months after an acute coronary syndrome. In the same trial, it was shown to be the only drug in its class that also reduced all-cause mortality without an excess of fatal bleeding. While the mechanism remains unclear, the benefits are real.

Q What are the disadvantages of using ticagrelor in comparison with other oral antiplatelet treatments?

Being more potent than clopidogrel, it has a higher incidence of minor and major bleeding.

Q Can you tell us about the ATLANTIC study? The ATLANTIC study was a double-blind, placebo-controlled trial designed to test the optimal timing of giving a second antiplatelet agent (ticagrelor) in patients undergoing PCI after an ST-elevation myocardial infarction (STEMI). It was a trial that was designed to test strategy (prehospital vs hospital administration of ticagrelor), not pharmacology.

\section{Q What were the inclusion criteria?}

All patients had a diagnosis of STEMI and planned to undergo primary PCI.

Q What were the primary end points?

The coprimary end points were:

- Percentage of patients not achieving ST segment resolution of at least $70 \%$ before PCI;
- The proportion of patients who did not have TIMI grade 3 flow in the infarct-related artery at initial angiography.

Q Can you summarize the main findings of the ATLANTIC study? What does this study add to the results from the PLATO trial \& how do you think the results will change the field?

With respect to the coprimary end points, this study showed no difference between prehospital and hospital administration of ticagrelor in patients undergoing primary PCI for STEMI. However, the strategy of giving prehospital ticagrelor was found to be safe and supports the European Society of Cardiology (ESC)/ European Association for Cardio-Thoracic Surgery (EACTS) 2014 guidelines on myocardial revascularization, which give a class I recommendation to starting dual antiplatelet therapy in STEMI patients on the first medical contact. There was a significant reduction in stent thrombosis at 30 days, which was a prespecified secondary end point, so there may be a potential benefit of prehospital administration of ticagrelor, but the incidence of stent thrombosis in the study was low overall. Interestingly, the incidence of stent thrombosis seen in the hospital-administered arm was similar to that seen in the PLATO trial.

The ATLANTIC study adds to the data from the PLATO trial and extends the potential use of ticagrelor to the prehospital setting in patients with STEMI.

\section{Q What are the future implications for the} use of ticagrelor? How do you anticipate its use in a clinical setting?

The ATLANTIC study will not change existing practice. Those centers and individuals who believe in early, prehospital administration of dual antiplatelet agents will see enough in the data to support their practice and be ESC/ EACTS guidelines compliant. They will use the potential reduction of early stent thrombosis as further supporting evidence. Those who prefer to continue using ticagrelor in hospital for this patient population can continue to do so on the basis that the primary end points were not met.

Q In your opinion, what has been the most exciting or influential finding in the field of cardiology in the last 5 years?

Undoubtedly, the answer must be the development of transcatheter aortic valve implantations 
for critical aortic stenosis. It has transformed the lives of patients blighted by a degenerative condition that reduces both quality and quantity of life.

Q In your opinion, what is the next hot topic in cardiology? What advances do you predict will be made? What are you most excited about?

This is a difficult question, given the amount and variety of research (imaging, leadless pacemakers, drugs and devices for heart failure and telemedicine, among many others) currently being undertaken in the world of cardiovascular diseases - all with the potential to revolutionize patient care. In pharmacology, I think current studies of novel anti-inflammatory agents and novel lipid-lowering agents have real potential to reduce and/or abolish the impact of coronary heart disease within our lifetime. From an interventionist's viewpoint, I think developments in percutaneous valve technologies will further reduce the need for open heart surgery to overcome dysfunctional valves whilst leadless pacemeakers have the potential to improve the lives of patients living long distances from cardiac centres. There are many more advances that are in early stages of development and justify the reason I chose cardiology as a career. The challenge, as ever, will be to make these advances affordable and available to the wider, global population - that, perhaps, is the greatest challenge that we all face.
Q Do you have any more comments to add with regards to the ATLANTIC study or ticagrelor?

A footnote to the main results was the interaction that was seen with morphine. Patients who received morphine in the ambulance derived no benefit from prehospital ticagrelor, whereas those not receiving the analgesic demonstrated significant benefits in the primary end point. While this is post hoc subgroup analysis, it supports preliminary data showing that patients given morphine have delayed gastric emptying and, in some cases, may vomit the ingested drugs. This has led to discussions on exploring the role of analgesia in patients with STEMI and, in particular, the need for morphine and its derivatives - watch this space.

\section{Disclaimer}

The opinions expressed in this interview are those of the interviewee and do not necessarily reflect the views of Future Medicine Ltd.

Financial \& competing interests disclosure

A Zaman has received honoraria for lectures and consultancy from Astra Zeneca. A Zaman has no other relevant affiliations or financial involvement with any organization or entity with a financial interest in or financial conflict with the subject matter or materials discussed in the manuscript apart from those disclosed.

No writing assistance was utilized in the production of this manuscript. 